\title{
Onchocerciasis: A Reality in Western Burkina Faso in 2016
}

Diallo $\mathbf{B}^{1,2^{*}}$, Konate $\mathbf{I}^{1,2}$, Andonaba JB ${ }^{1,2}$, Sangaré $\mathbf{I}^{1,3}$, Konsegree $\mathbf{V}^{1,4}$, Traoré/Dolo $\mathbf{M}^{1,5}$ and Bamba $\mathbf{S}^{1,3}$

${ }^{1}$ Higher Institute of Health Sciences, Polytechnic University of Bobo-Dioulasso, Burkina Faso

${ }^{2}$ Department of Dermatology-Venereology, CHU Sourô Sanou, Bobo-Dioulasso

${ }^{3}$ Service of Parasitology-Mycology, CHU Sourô Sanou, Bobo-Dioulasso

${ }^{4}$ Department of Pathological Anatomy, Sourô Sanou Hospital, Bobo-Dioulasso

${ }^{5}$ Department of Ophthalmology, CHU Sourô Sanou, Bobo-Dioulasso

"Corresponding author: Diallo B, Higher Institute of Health Sciences, Polytechnic University of Bobo-Dioulasso, Burkina Faso, Tel: (+226) 70114900; E-mail: bkrdiallo@yahoo.fr

Received date: November 10, 2016; Accepted date: November 19, 2016; Published date: November 30, 2016

Copyright: (c) 2016 Diallo B, et al. This is an open-access article distributed under the terms of the Creative Commons Attribution License, which permits unrestricted use, distribution, and reproduction in any medium, provided the original author and source are credited.

\begin{abstract}
Sporadic cases of onchocerciasis continue to be registered after intensive campaigns under the aegis of the Onchocerchiasis Control program (OCP) that led to the control of the disease in Burkina Faso since 2010. It is now part of the neglected tropical diseases (NTDS). We report 3 recent confirmed cases at the Teaching Hospital of Bobo-Dioulasso, that are worrying in this context.

There were two men and a woman, all coming from the west area of Burkina Faso, border with Ivory Coast and Ghana, and they have intermittent stays in a side and other of the two borders.

The reason for consultation was a chronic pruritus evolving since 2 to 4 years. The examination noted dermatological damages combining in a variable way, painless and mobile nodules localized at the trochanters and chest seats, a leuco-melanodermia of the legs or polymorph chronic prurigo lesions, without nodules. The diagnosis of onchoceriasis was confirmed by presence of blood hyper-eosinophilia, the presence of many Onchocerca volvulus micofilariaes in the dermal juice and many adult worms in histology.
\end{abstract}

Because that Onchocerciasis became quite rare in everyday practice, the statement of 3 hospital cases collected in 8 months is worrying because the vector and the parasite are still present in the affected areas, with a possible transmission. So, a new outbreak is to fear, especially with the internal and cross-border population mobility.

Keywords: Onchocerciasis; Neglected tropical disease; Treatment with ivermectin under community guidelines; Burkina Faso

\section{Introduction}

Onchocerciasis is a parasitic dermatosis caused by Onchocerca volvulus, transmitted by gnats breeding in fast-flowing streams: stimulia. Adult worms live 10-15 years in subcutaneous nodules and produce embryos called microfilaria that invade the dermis and tissues of the eye.

They constitute the pathogenic stage of the parasite. Most of the manifestations of onchocerciasis are due to a cellular immune response against microfilariae and against symbiotic bacteria (Wolbachia) present inside the parasite.

The symptoms increase in intensity with the parasite load [1]. Severe lesions can lead to blindness, hence the term "river blindness" used to describe this disease.

Sporadic cases of onchocerciasis continue to be recorded after extensive control campaigns under the aegis of the Onchocerchiasis Control Program (OCP), which resulted in control of the disease in Burkina Faso in 2010 [2].
It is now part of Neglected Tropical Diseases (NTD). We report 3 recent confirmed cases at the Bobo-Dioulasso University Hospital, a source of concern in this context.

\section{Case Presentation}

Case 1: In May 2015, Mr. SM, 52, was referred to us from the Medical Center of Orodara for a generalized pruritus evolving during 4 years. He had spent several years in Cote d'Ivoire and had returned to settle in Kénédougou for 2 years.

Dermatological examination noted subcutaneous, painless, hard, mobile, lateral-thoracic seat and trochanter nodules (Figure 1) and hypochromic macules in drops, grouped in pre-tibial placards constituting the Classical leucomelanodermy of the legs (Figure 2). Ophthalmologic examination was normal.

On the paraclinic level, $24.1 \%$ blood eosinophilia was observed, the presence of numerous mobile microfilariae of Onchocerca volvulus in dermal juice (Figure 3 ) and adult worms in the histology of a nodule. 


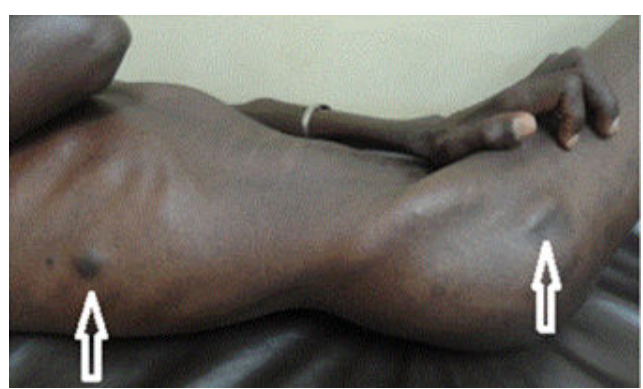

Figure 1: Onchocerciasis: nodules subcutaneous, painless, hard, mobile, latero-thoracic seat and facing trochanter.

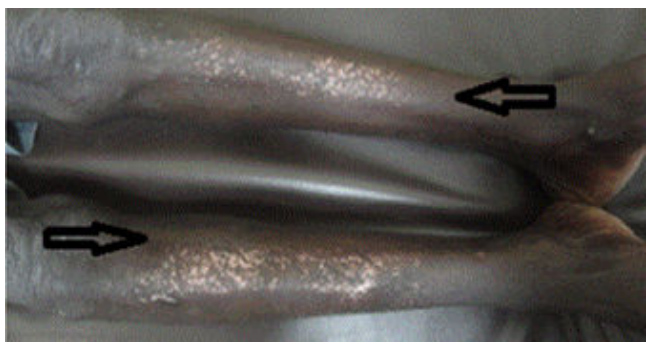

Figure 2: Hypochromic macules in drops, grouped in pre-tibial placards constituting the leucomelanodermy of the legs during an onchocerciasis.

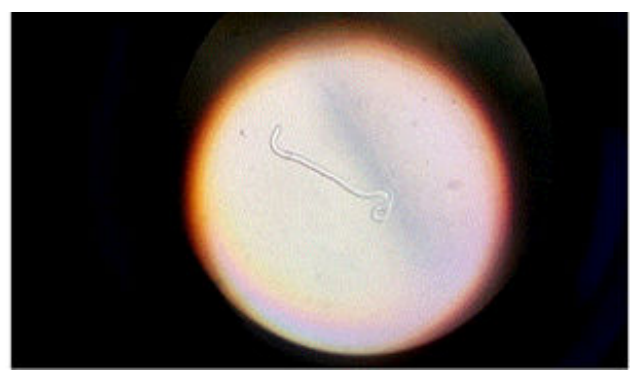

Figure 3: Hypochromic macules in drops, grouped in pre-tibial placards constituting the leucomelanodermy of the legs during an onchocerciasis.

Case 2: Ms. TN, 53, a native of Mondon, a village in Banfora, but residing in Aboisso, Côte d'Ivoire for more than 10 years with periodic visits to her home town, consulted in August 2015 for Subcutaneous nodules trochanteric, painless, mobile, evolving for 3 years. The biopsy of one of these nodules confirmed the diagnosis of onchocerciasis by the presence of adult wires (Figure 4).

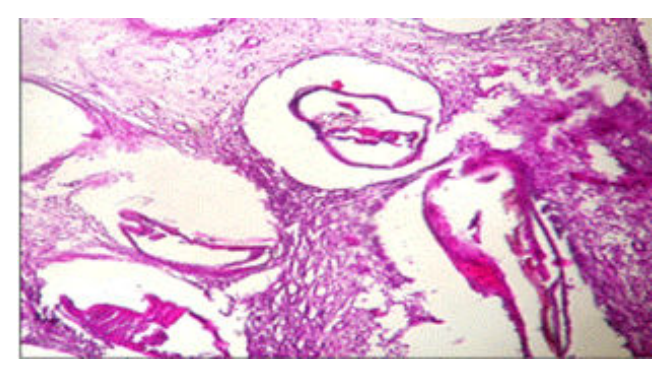

Figure 4: Wires to the histology of a subcutaneous nodule biopsy, confirming the diagnosis of onchocerciasis.

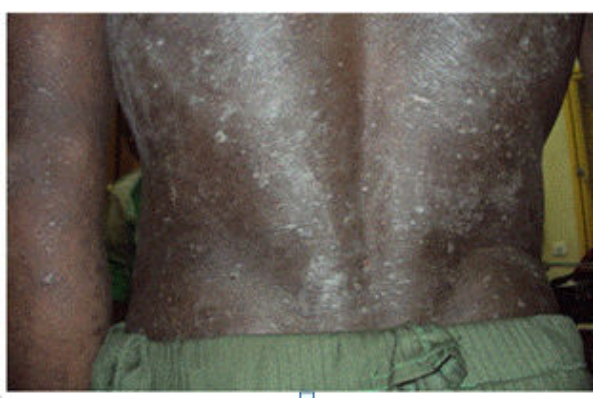

Figure 5: Polymorphic lesions made of scratch striations, smal papules, excoriations, recent hypochromic scar lesions and hyperpigmented old scars.

Case 3: $\mathrm{Mr} \mathrm{DH}, 32$, from Koueré (Banfora district), with intermittent stays in Ghana, consulted in January 2016 for diffuse pruritus evolving for 2 years. He says he did not receive the mass treatment with ivermectin and albendazole. On examination there were no nodules but polymorphic lesions of chronic prurigo disseminated throughout the integument, made of scratch striations, small papules, excoriations, recent hypochromic scar lesions and hyperpigmented old scars (Figure 5). The smear of the dermal juice showed rare microfilariae of Onchcerca volvulus and the NFS a eosinophyll at $22 \%$.

Burkina Faso was once a highly endemic country with onchocerciasis, with prevalences of up to $60 \%$ to $80 \%$ in 1974 and a blindness rate of up to $10 \%$ in some hyperendemic villages [2]. The onchocerciasis control campaigns carried out under the auspices of the Onchocerchiasis Control Program (OCP) resulted in control of the disease in 2010 and are no longer a public health problem in Burkina Faso, but the disease is not eradicated and is the subject of longitudinal entomo-epidemiological surveillance. In its strategic plan to combat NTD 2012-2016, the Ministry of Health has set itself the goal of eliminating onchocerciasis by 2020 by controlling its transmission in the 6 remaining endemic districts in order to avoid any increase in onchocerciasis disease.

All of our cases were from the western area of Burkina Faso, bordering Côte d'Ivoire and Ghana, which is one of the problem districts where local onchocerciasis persisted in some villages in 2010 [2]. 
Citation: Diallo B, Konate I, Andonaba JB, Sangaré I, Konsegree V, et al. (2016) Onchocerciasis: A Reality in Western Burkina Faso in 2016. J Trop Dis 4: 225. doi:10.4172/2329-891X.1000225

Page 3 of 3

These typical onchocerciasis tables described above should be of particular interest to health professionals and dermatologists, who should not misunderstand the diagnosis of this neglected tropical disease which has become rare but not yet eradicated. The diagnosis is simple enough to think of it before a chronic prurigo with polymorphic lesions (scratching lesions, papules, lichenfications, cutaneous atrophy and localized depigmentation at the tibial crests) or before painless, hard and mobile subcutaneous nodules. Most often located opposite the bone planes. Confirmatory diagnosis is based on evidence of microfilariae in a bleeding cutaneous biopsy and / or on the histology of the subcutaneous nodule removal that will show adult worms.

Since onchocerciasis has become rather rare in everyday practice, the observation of 3 hospital cases collected in 8 months is worrying because the parasite is always present in the areas concerned, thus a possible transmission. A re-emergence is therefore to be feared, especially with the internal and cross-border mobility of populations [3]. Epidemiological and entomological assessments are needed during this phase to ensure that there is no increase in the parasite population and transmission.

The strategy for the elimination of this endemic is essentially based on the treatment with ivermectin integrated into the health system (Treatment with Ivermectin under Community Directives) [4].

African studies have provided evidence that elimination by Ivermectin treatment was feasible in endemic foci $[1,3]$, this treatment should be done regularly and for a long period of time because of the longevity of adult worms Effect of the drug on the last [5,6]. Most adult worms re-start producing microfilariae a few weeks after treatment, causing a rise in microfilarial loads, hence the need for repeated catches every six months or every year (in mass treatment.

\section{Conclusion}

The threat of onchocerciasis is still present in the Comoé basin, west of Burkina Faso. This means the need for clinical and biological monitoring and the continuation or even intensification of Ivermectin Treatment under Community Directives.

\section{References}

1. World Health Organization: African Program for Onchocerciasis Control (WHO/APOC) (2010).

2. Ministry of Health of Burkina Faso, Directorate of Disease Control (2012) Strategic Plan for the Control of Neglected Tropical Diseases in Burkina Faso.

3. Gustavsen K, Sodahlon Y, Bush S (2016) Cross-Border Collaboration for Neglected Tropical Diseases: Lessons Learned from Onchocerciasis Control and Elimination in the Mano River Union (West Africa). Globalization and Health 12: 44.

4. (2016) WHO. Guidelines for Stopping Mass Drug Administration and Verification Elimination of Human Onchocerciasis: criteria and procedures. Geneva: World Health Organization.

5. Badji AB (2001) From the Control of Onchocerciasis to Human Ecology: Methods of Control and Post Programs in West Africa. Memory of the International Certificate of Human Ecology, University of Bordeaux.

6. Senyonjo L, Oye J, Bakajika D, Biholong B, Tekle A, et al. (2016) Factors Associated with Ivermectin Non-Compliance and its Potential Role in Sustaining Onchocerca volvulus Transmission in the West Region of Cameroon. PLoS Negl Trop Dis 10: 0004905. 